\title{
Erratum: Filling-Factor-Dependent Magnetophonon Resonance in Graphene [Phys. Rev. Lett. 99, 087402 (2007)]
}

\author{
M. O. Goerbig, J.-N. Fuchs, K. Kechedzhi, and Vladimir I. Fal'ko \\ (Received 18 September 2009; published 21 October 2009)
}

DOI: 10.1103/PhysRevLett.103.179901

PACS numbers: 78.30.Na, 73.43.-f, 81.05.Uw, 99.10.Cd

We have underestimated the numerical value of the mode splitting of the magnetophonon resonance [see the paragraph after Eq. (5) in our Letter [1] ] by a factor of 2. This is a result of two mistakes. First, there is a factor of $\sqrt{2}$, which finds its origin in an erroneous normalization of the circular polarized phonons. They should indeed be defined as $u_{\circlearrowleft}=\left(u_{x}+\right.$ $\left.i u_{y}\right) / \sqrt{2}$ and $u_{\circlearrowright}=\left(u_{x}-i u_{y}\right) / \sqrt{2}$ [and not as $u_{\circlearrowleft}=u_{x}+i u_{y}$ and $u_{\circlearrowright}=u_{x}-i u_{y}$ as incorrectly assumed on page 1 , second column], such that the associated phonon operators $b_{\mathcal{A}}$ obey the usual commutation relations $\left[b_{\mathcal{A}}, b_{\mathcal{A}^{\prime}}^{\dagger}\right]=\delta_{\mathcal{A} \text {, } \mathcal{A}^{\prime}}$, with $\mathcal{A}=\cup, \cup$. This yields a factor of $\sqrt{2}$ in the definition of the effective coupling constants [Eq. (3)], which read in the corrected form

$$
g_{\cup}(n)=g \sqrt{\left(1+\delta_{n, 0}\right) \gamma} \sqrt{\bar{\nu}_{-,(n+1)}-\bar{\nu}_{+, n}}, \quad g_{\circlearrowright}(n)=g \sqrt{\left(1+\delta_{n, 0}\right) \gamma} \sqrt{\bar{\nu}_{-, n}-\bar{\nu}_{+,(n+1)}} .
$$

As a consequence, the zero-field dimensionless coupling constant $\lambda$ [defined in the first column of page 3 ] is multiplied by a factor of 2 and becomes $\lambda=(2 / \sqrt{3} \pi)(g / t)^{2}$.

Second, we also underestimated the numerical value of the electron-phonon coupling constant $g$ by a factor of $\sqrt{2}$. Indeed, $g$ defined in our work [see Eq. (1)] is related to $\left\langle g_{\Gamma}^{2}\right\rangle_{F} \simeq 0.0405 \mathrm{eV}^{2}$ computed by Piscanec et al. [2] as $g=$ $\sqrt{2\left\langle g_{\Gamma}^{2}\right\rangle_{F}} \simeq 0.28 \mathrm{eV}$ and not as $g=\sqrt{\left\langle g_{\Gamma}^{2}\right\rangle_{F}} \simeq 0.2 \mathrm{eV}$ as incorrectly assumed in our Letter. As a consequence the dimensionless coupling constant becomes $\lambda \simeq(3.3$ to 4$) \times 10^{-3}$ [instead of $\lambda \simeq 10^{-3}$ ] if the hopping amplitude $t$ is taken in between 2.7 and $3 \mathrm{eV}$.

As a result of the two factors of $\sqrt{2}$, the numerical estimate for the mode splitting $2 g_{\mathcal{A}}$ at $\nu=0$ and $B \simeq 30 \mathrm{~T}$ [at the discussed resonance,$- 1 \Rightarrow 0$, and $0 \Rightarrow+, 1$, see second column of page 3] becomes $2 g_{\mathcal{A}} \sim 16 \mathrm{meV}\left(\sim 130 \mathrm{~cm}^{-1}\right)$ [instead of $2 g_{\mathcal{A}} \sim 8 \mathrm{meV}$ ]. The effect is therefore twice larger than initially predicted. The conclusions of our work remain unaltered.

In addition, recent measurements [3,4] find larger values of the dimensionless coupling constant $\lambda=4.4 \times 10^{-3}$ and $5.3 \times 10^{-3}$, respectively, which correspond to an even larger mode splitting of the predicted magnetophonon resonance $2 g_{\mathcal{A}} \sim 20 \mathrm{meV}\left(\sim 160 \mathrm{~cm}^{-1}\right)$.

We would like to thank C. Faugeras and M. Potemski for having drawn our attention to the underestimated value of the mode splitting. See also their recent paper where they measure the magnetophonon resonance [5].

[1] M. O. Goerbig, J.-N. Fuchs, K. Kechedzhi, and Vladimir I. Fal'ko, Phys. Rev. Lett. 99, 087402 (2007).

[2] S. Piscanec, M. Lazzeri, F. Mauri, A. C. Ferrari, and J. Robertson, Phys. Rev. Lett. 93, 185503 (2004).

[3] S. Pisana, M. Lazzeri, C. Casiraghi, K. S. Novoselov, A. K. Geim, A. C. Ferrari, and F. Mauri, Nature Mater. 6, 198 (2007).

[4] J. Yan, Y. Zhang, P. Kim, and A. Pinczuk, Phys. Rev. Lett. 98, 166802 (2007).

[5] C. Faugeras, M. Amado, P. Kossacki, M. Orlita, M. Sprinkle, C. Berger, W. A. de Heer, and M. Potemski, arXiv:0907.5498 [Phys. Rev. Lett. (to be published)] 